\title{
Hasmer Koyununda Arteria Celiaca ve Dallarının Makroanatomik İncelenmesi
}

\author{
Derviş ÖZDEMiR $^{1 \mathrm{a} \otimes}, Z^{2}$,ekeriya ÖZÜDOĞRU ${ }^{2 b}$, Hülya BALKAYA ${ }^{1 \mathrm{c}}$, Hülya KARA $^{1 \mathrm{~d}}$
}

1. Atatürk Üniversitesi, Veteriner Fakültesi, Anatomi Anabilim Dalı, Erzurum, TÜRKiYE.

2. Aksaray Üniversitesi, Veteriner Fakültesi, Anatomi Anabilim Dalı, Aksaray, TÜRKiYE.

ORCID: 0000-0002-6038-0485 ${ }^{\mathrm{a}}, 0000-0002-07893628^{\mathrm{b}}, 0000-0001-6164-158 \mathrm{X}^{\mathrm{c}}, 0000-0002-7678-6471^{\mathrm{d}}$

\begin{tabular}{|c|c|c|}
\hline $\begin{array}{c}\text { Geliş Tarihi/Received } \\
\text { 23.12.2019 }\end{array}$ & $\begin{array}{c}\text { Kabul Tarihi/Accepted } \\
\text { 05.02.2020 }\end{array}$ & $\begin{array}{c}\text { Yayın Tarihi/Published } \\
27.10 .2020\end{array}$ \\
\hline $\begin{array}{l}\text { Bu makaleye atıfta bulunmak } \\
\text { Özdemir D, Özüdoğru Z, Balka } \\
\text { Atatürk Üniversitesi Vet. Bil. D }\end{array}$ & $\begin{array}{l}\text { ticle: } \\
\text { her Koyununda Arteria } \\
\text { 5, 2020. DOI: 10.17094/ }\end{array}$ & In Makroanatomik İncelenmesi. \\
\hline
\end{tabular}

Öz: Bu çalışmada, hasmer koyun ırkının arteria celiaca ve dallarının makro-anatomik olarak incelenmesi amaçlandı. Araştırmada altı adet hasmer koyunu kullanıldı. Karın boşluğu açılan materyallere, aorta abdominalis'ten renklendirilmiş latex ve monopolymethymethacrylate (takilon) verildi. Aorta abdominalis'in cavum abdominis'te verdiği ilk dalın arteria celiaca olduğu belirlendi. Arteria celiaca'dan önce arteria lienalis'in daha sonra arteria hepatica ile arteria gastrica sinistra'nın ortak bir kök halinde ayrıldığı tespit edildi. Arteria lienalis'in; rami pancreatici, ramus epiploicus, arteria ruminalis sinistra ve arteria ruminalis dextra isimli dallara sahip olduğu gözlendi. Arteria hepatica'nın, porta hepatis seviyesinde arteria cystica, arteria gastrica dextra ve rami pancreatici'yi verdiği ve damarın devamının arteria gastroduodenalis olduğu belirlendi. Arteria celiaca'nın devamı durumunda olan arteria gastrica sinistra'nın, en kalın dal olduğu, seyri esnasında arteria reticularis'i, arteria gastroepiploica sinistra ile arteria reticularis accessoria'yı verdiği ve daha sonra arteria gastrica dextra ile anastomoz yaparak sonlandığı tespit edildi. Arteria reticularis, ramus ruminalis ve ramus reticularis isimli dallara ayrılmaktaydı. Arteria reticularis accessoria'nın ise reticulum'un visceral yüzü ile omasum'un parietal yüzüne birkaç dal vererek sonlandığı belirlendi. Sonuç olarak; hasmer koyun ırkında arteria celiaca'nın aorta abdominalis'teki başlangııından, organları vaskülarize eden dallarına kadar birtakım farklılıklara sahip olduğu belirlendi. Tespit edilen farklılıkların ırk özelliğinden kaynaklanabileceği kanısına varıldı.

Anahtar Kelimeler: Anatomi, Arteria celiaca, Hasmer koyunu.

\section{Macroanatomic Investigation of Arteria Celiaca and Its Branches in Hasmer Sheep}

\begin{abstract}
In this study, it was aimed to investigate the arteria celiaca and its branches macro-anatomically. Six hasmer sheep were used in the study. Latex and monopolymethymethacrylate (tachilon) colored to abdominal aorta were given to the abdominal cavity. It was determined that the first branch of aorta abdominalis in the cavum abdominis was arteria celiaca. Arteria lienalis before arterial celiaca and then arteria hepatica and arteria gastrica sinistra were separated into a common root. Arteria lienalis branched rami pancreatici, ramus epiploicus, arteria ruminalis sinistra and arteria ruminalis dextra. Arteria hepatica gave arteria cystica, arteria gastrica dextra and rami pancreatici at the porta hepatis level and the continuation of the vessel was arteria gastroduodenalis. The arteria gastrica sinistra, which is the continuation of the arterial celiaca, was found to be the thickest branch, during which it gave arteria reticularis, arteria gastroepiploica sinistra and arteria reticularis accessoria, and then ended up with anastomosis with arteria gastrica dextra. Arteria reticularis, ramus ruminalis and ramus reticularis were divided into branches. Arteria reticularis accessoria was terminated by giving a few branches to the visceral face of the reticulum and the parietal face of the omasum. As a result; in the hasmer sheep breed, the origin of the artery celiaca in the aorta abdominalis and vascularizing branches of the organs were determined to have several differences. It was concluded that the differences could be due to race characteristics.
\end{abstract}

Keywords: Anatomy, Arteria celiaca, Hasmer sheep.

\footnotetext{
区erviş Özdemir

Atatürk Üniversitesi, Veteriner Fakültesi, Anatomi Anabilim Dalı, Erzurum, TÜRKiYE.

e-posta: dozdemir@atauni.edu.tr
} 
Giriş

$\mathrm{T}$ ürkiye'de düşük verimli yerli ırkları geliştirmek ve tekstildeki hammadde ihtiyacını karşılamak amacıyla, et ve yapağıya yönelik koyun ırkları ıslah çalışmaları planlanmıştır. Günümüz tekstil sanayisinde suni elyafın daha çok kullanılması ve koyunculuk alanındaki getirilerin büyük bir kısmını et veriminin teşkil etmesi nedeniyle, Türkiye'de de etçi koyun ırkı geliştirilmesi çalışmaları başlatılmıştır. Bu amaçla, 1980'li yıllardan sonra yurtdışından İngiliz ve Alman etçi koyun ırklarının koçları ithal edilmiş ve bu ırklar yerli ırklarla melezlenerek kasaplık kuzular yetiştirilmiştir $(1,2)$. Bu melez ırklardan biri olan hasmer koyunu, Hampshire, Alman Siyah Başlı ve Merinos koyun ırklarının üçlü melezlemesiyle elde edilen bir koyun ırkıdır (3).

Koyun (4-14), keçi $(5,7,15-19)$ ve bazı memeli türlerinde (20-24) arteria celiaca anatomik olarak araştııımıştır. Ancak, Türkiye'de yerli bir ırk olarak bulunan Hasmer koyun ırkının arteria celiaca ve dalları hakkında herhangi bir çalışmanın bulunmadığı tespit edilmiş ve bu araştırmayla konu hakkındaki eksikliğin giderilmesi amaçlanmıştır.

\section{MATERYAL ve METOT}

Çalışmada, Konya Bahri Dağdaş Uluslararası Tarımsal Araştırma Enstitüsü Müdürlüğü, Deney Hayvanları Ünitesi Birimi'nden tedarik edilen altı adet hasmer koyunu kullanıldı. Aorta abdominalis içerisine $\% 10^{\prime}$ luk tuzlu su verilerek yıkandıktan sonra, kırmızı kumaş boyası ile latex karıştırılarak damar içine enjekte edildi. Materyaller \%10'luk formaldehit solüsyonunda 24 saat süre ile bekletildi ve diseke edildi. Diseksiyonları tamamlanan materyallerin, Nikon P900 83X Optik Zoom marka fotoğraf makinesi ile fotoğrafları alındı. Terminolojide Nomina Anatomica Veterineria (N.A.V.) esas alındı (25). Çalışma için gerekli izin, Atatürk Üniversitesi Veteriner Fakültesi Birim Etik Kurul'undan alındı (Tarih: 14.05.2018, sayı: 2018/55).

\section{BULGULAR}

Aorta abdominalis'in, hiatus aorticus aracılığı ile cavum thoracis'ten cavum abdominis'e geçtiği gözlendi. Cavum abdominis'te verdiği ilk dal olan arteria celiaca'nın birinci lumbal omur hizasında ventrale doğru seyrettiği belirlendi.

Arteria celiaca'dan ilk ayrılan damarın arteriae phrenicae caudales olduğu gözlendi. Daha sonra sırasıyla arteria lienalis ve arteria hepatica ile arteria gastrica sinistra'nın ortak bir kök halinde orijin aldığı tespit edildi (Şekil 1).

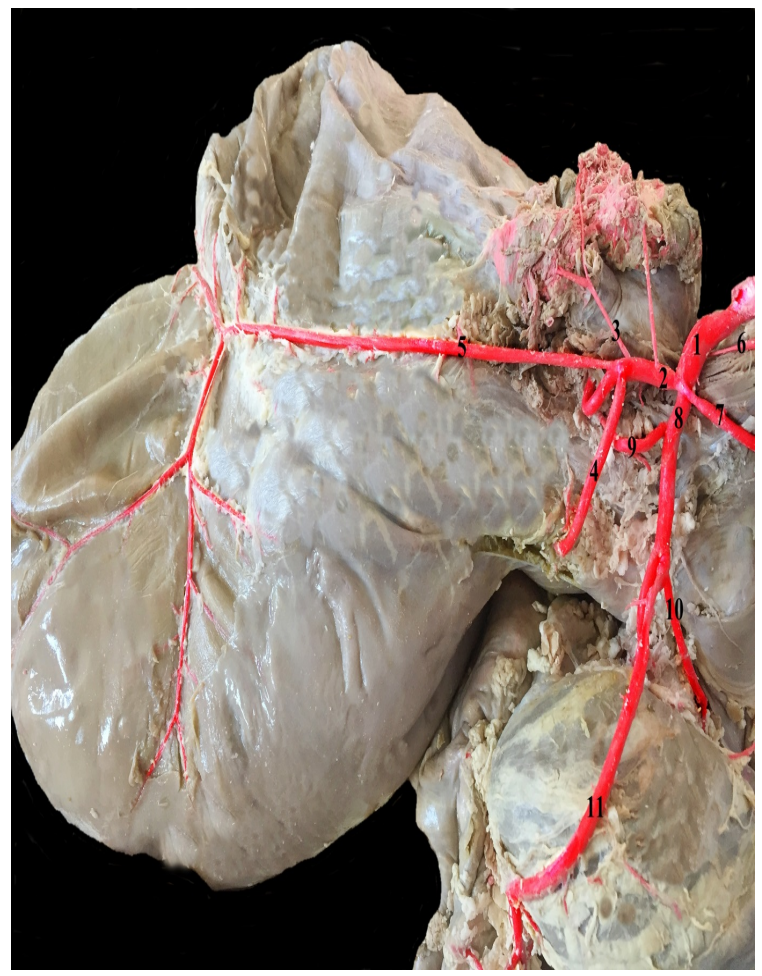

Şekil 1. Arteria lienalis ve arteria gastrica sinistra'nın dalları.

Figure 1. Arteria lienalis and arteria gastrica sinistra's branches.

1-a. celiaca, 2- a. lienalis, 3- ramus epiploicus, 4- a. ruminalis dextra, 5-a. ruminalis sinistra, 6-a. phrenica caudalis, 7- a. hepatica, 8- a. gastrica sinistra, 9- a. reticularis, 10- a. reticularis accessoria, 11- a. gastroepiploica sinistra.

\section{Arteria lienalis}

Arteria lienalis, pancreas'a giden rami pancreatici'yi verdikten sonra omentum majus içinde ramus epiploicus ve arteria ruminalis sinistra ve arteria ruminalis dextra'yı verdikten sonra arteria lienalis olarak hilus lienalis'e giren damarın iki dala ayrıldığı görüldü (Şekil 1,2). 


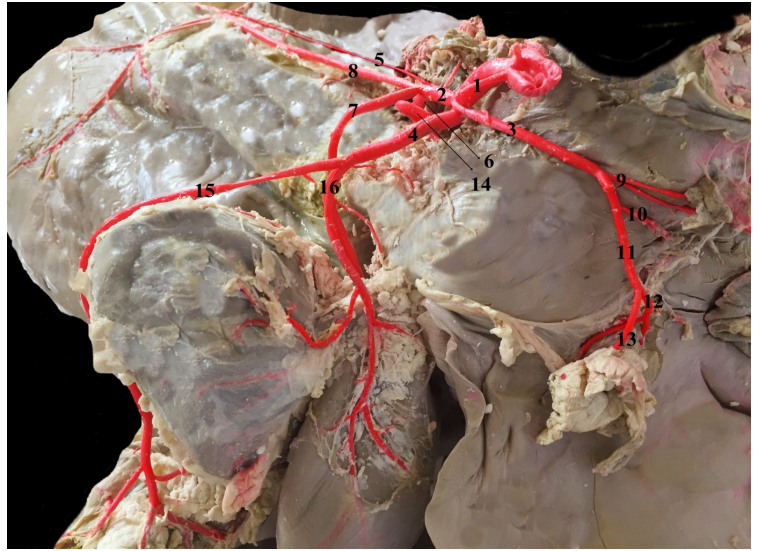

Şekil 2. Arteria celiaca ve dalları.

Figure 2. Arteria celiaca's branches.

1- a. celiaca, 2- a. lienalis, 3- a. hepatica, 4- a. gastrica sinistra, 5- ramus epiploicus, 6- rami pancreatici, 7- a. ruminalis sinistra, 8- a. ruminalis dextra, 9- ramus pancreaticus, 10- a. cystica, 11-a. gastroduodenalis, 12 a. pancreaticoduodenalis cranialis, 13- a. gastroepiploica dextra, 14- a. reticularis, 15- a. gastroepiploica sinistra, 16- a. reticularis accessoria.

\section{Arteria ruminalis sinistra}

Arteria lienalis'in sol tarafından orijin alan arteria ruminalis sinistra (Şekil 1,2), caudoventral seyrederek atrium ruminis'in sağ yüzüne ulaştığı gözlendi. Damar'ın daha sonra rumen'in sulcus cranialis'inden geçen sulcus longitudinalis sinister içerisindeki seyrinde saccus cecus craniodorsalis ile saccus cecus cranioventralis'in parietal yüzünü vaskülarize eden dallar verdiği belirlendi.

\section{Arteria ruminalis dextra}

Arteria ruminalis dextra'nın (Şekil 1,2), arteria lienalis'in sağ tarafından orijin aldığı ve caudoventral bir seyirle rumenin visceral yüzüne ulaştığı tespit edildi. Sulcus longitudinalis dexter'in içerisinde seyreden arteria ruminalis dexter'in, sulcus caudalis'e kadar uzandığı ve bu oluktan rumen'in parietal yüzüne geçerek sonlandığı belirlendi. Seyri sırasında rumen'in parietal ve visceral yüzünde saccus cecus'ları vaskülarize eden damarlar verdiği gözlendi.

\section{Arteria hepatica}

Arteria gastrica sinistra ile ortak kök halinde arteria celiaca'dan orijin alan arteria hepatica'nın, karaciğerin visceral yüzünden porta hepatis'e doğru seyrettiği ve porta hepatis seviyesinde arteria cystica, arteria gastrica dextra ile rami pancreatici isimli damarları verdikten sonra arteria gastroduodenalis olarak seyrine devam ettiği saptandı (Şekil 2). Arteria gastroduodenalis'in, arteria pancreaticoduodenalis cranialis'i verdikten sonra arteria gastroepiploica dextra ismini aldığı ve abomasum'un curvatura minor'unda arteria gastroepiploica sinistra ile anastomoz yaptığı tespit edildi.

\section{Arteria cystica}

Arteria hepatica'dan ayrıldıktan sonra karaciğerin visceral yüzünde seyreden arteria cystica'nın, vesica fellea'ya dağıldığı belirlendi (Şekil 2).

\section{Arteria gastrica dextra}

Karaciğerin visceral yüzünde seyreden arteria gastrica dextra'nın (Şekil 2), karaciğerin caudal lobu için arteria lobi caudati'yi verdikten sonra arteria gastrica sinistra ile anastomoz yaptığı tespit edildi. Seyri sırasında abomasum'un curvatura minor'u üzerindeki seyrinde omentum minus ve bu kenarı besleyen dallar verdiği görüldü.

\section{Arteria gastroduodenalis}

Arteria hepatica'nın devamı olarak görülen arteria gastroduodenalis'in, arteria pancreatico duodenalis cranialis'i verdiği ve abomasum'un curvatura major'u üzerinde arteria gastroepiploica dextra ismini aldığı gözlendi (Şekil 2). Arteria gastroepiploica dextra'nın arteria gastroepiploica sinistra ile anastomoz yaparak sonlandığı ve damarın seyri sırasında abomasum'un curvatura major isimli kenarını ve omentum majus'u vaskülarize ettiği belirlendi.

\section{Arteria pancreaticoduodenalis cranialis}

Arteria gastroduodenalis'ten orijin alan arteria pancreaticoduodenalis cranialis'in (Şekil 2), duodenum'un pars cranialis kısmında, duodenum ve 
pancreas'ı besleyen dallar verdiği gözlendi. Arteria mesenterica cranialis'in bir dalı olan arteria pancreaticoduodenalis caudalis ile anastomoz yaptığı görüldü.

\section{Arteria gastrica sinistra}

Arteria hepatica ile ortak bir kök halinde arteria celiaca'dan orijin alan arteria gastrica sinistra'nın, damarın devamı durumunda olduğu gözlendi. Arteria celiaca'nın verdiği dalların en kalını olmasıyla dikkat çekmekteydi. Bu damarın seyri esnasında ilk olarak arteria reticularis'i verdiği, omasumla reticulum arasındaki alanda ise arteria gastroepiploica sinistra ile arteria reticularis accessoria'yı verdikten sonra omasum'un visceral yüzünde sağlı ve sollu dallar vererek seyrine devam ettiği belirlendi (Şekil 1,2). Abomasum'un curvatura minor isimli kenarında arteria gastrica dextra ile anastomoz yaparak sonlandığı tespit edildi.

\section{Arteria reticularis}

Arteria gastrica sinistra'dan orijin alan arteria reticularis'in (Şekil 1,2), sulcus reticuloruminalis içerisindeki seyrinde ramus ruminalis ve ramus reticularis isimli dalları verdiği gözlendi.

\section{Arteria gastroepiploica sinistra}

Atrium ruminis üzerinde arteria gastrica sinistra'dan cranioventral yönde orijin alan arteria gastroepiploica sinistra'nın (Şekil 1,2), sulcus reticulo-omasi'den geçerek abomasum'un curvatura major adlı kenarında arteria gastroepiploica dextra ile anastomoz yaptığı ve seyri sırasında ve omentum majus, reticulum, omasum ve abomasum'a dallar verdiği tespit edildi.

\section{Arteria reticularis accessoria}

Arteria gastroepiploica sinistra'dan hemen sonra arteria gastrica sinistra'dan orijin alan arteria reticularis accessoria'nın (Şekil 1,2), reticulumun visceral yüzü ile omasum'un parietal yüzünde birtakım dallar verdiği belirlendi.

\section{TARTIŞMA ve SONUÇ}

Hiatus aorticus aracılığı ile cavum thoracis'ten cavum abdominis'e geçen aorta abdominalis'in, cavum abdominis'te verdiği ilk dalın arteria celiaca olduğu tespit edildi. Arteria celiaca'nın birinci lumbal omur hizasında ventrale doğru seyrettiği belirlendi. Arteria celiaca'nın orijini ve aorta abdominalis'in ilk dalı olduğu şeklindeki bulgular, bazı koyun $(9,10)$ ve keçi ırklarında da $(17,18)$ saptanmıştır. Özüdoğru ve ark. (4) Konya merinosunda; Dursun (26) ve Türkmenoğlu (7) ruminantta arteria celiaca'nın son thoracal ve birinci lumbal omur arasından orijin aldığını bildirirken, Alsafy (15) ile El Gendy (16) keçide, 1 . ve 2 . lumbal omur düzeyinde aorta abdominalis'in ventralinden orijin aldığını rapor etmişlerdir. Bazı araştırmacılar $(5,6,8,15,20)$ ise materyallerin bir kısmında arteria celiaca'nın, arteria mesenterica cranialis ile ortak bir kök halinde aorta abdominalis'ten orijin aldığını bildirmişlerdir.

Özüdoğru ve ark. (4), Türkmenoğlu (7) materyal olarak kullandığı koyunların üç tanesinde arteria hepatica ile arteria gastrica sinistra'nın ortak bir kök halinde çıktığını ve Maala ve ark. (19) keçide arteria celiaca'nın dallanmasının altı değişik şekilde olduğunu ve bunlardan birisinde arteria gastrica sinistra ile arteria hepatica'nın ortak bir kök şeklinde çıktığını bildirmişlerdir. Bu çalışmada da arteria gastrica sinistra ile arteria hepatica'nın ortak bir kök olarak arteria celiaca'dan orijin aldığı tespit edildi. Bazı yazarlar $(5,6,8,21,27,28)$ ise arteria celiaca'dan; arteria lienalis, arteria gastrica sinistra ve arteria hepatica'nın ayrı üç dal olarak çıktığını ifade etmişlerdir. Bazı araştırmacılar da $(10,15,24)$ arteria celiaca'dan; arteria lienalis, arteria hepatica, arteria gastrica sinistra ve arteria ruminalis sinistra olmak üzere dört ayrı dal olarak ayrıldıklarını belirtmişlerdir.

A. lienalis'in; ramus epiploicus, arteria ruminalis sinistra ve arteria ruminalis dextra'yı verdikten sonra arteria lienalis olarak dalakta sonlandığı tespit edildi. Benzer bulguların koyun (10), keçi (15) ve buffaloda (20) da gözlendiği belirtilmiştir. 
Arteria gastrica sinistra'nın arteria celiaca'nın verdiği en kalın dal olduğu ve aynı zamanda damarın devamı niteliği taşıdığı şeklindeki tespit, Boccaletti ve Borelli (11) ile Mohamed ve ark. (10) ile koyunda, Mohamed ve ark. (17)'nın keçide tespit ettikleri bulgularla uyumludur.

Literatürde (26) arteria hepatica'nın arteria gastrica sinistra'dan daha kalın bir damar olduğu bildirilmiştir. Bu çalışmada da arteria hepatica'nın arteria gastrica sinistra'dan daha ince bir damar olduğu belirlenmiştir.

Arteria cystica'nın, keçi (15), ruminant ve sus'ta (29) arteria hepatica'dan başlangıç aldıktan sonra karaciğerin visceral yüzünde seyrettiği ve vesica fellea'ya kadar uzandığı şeklindeki bildirim araştırma bulgularıla benzerdir.

Rumen'in atrium ruminis ile reticulum'un caudal yüzünü besleyen arteria reticularis'in, arteria gastrica sinistra'dan orijin aldığı belirlenmiştir. Nayar ve ark. (30) keçide, Türkmenoğlu (7) koyunda, Smuts ve Bezuidenhout (28) devede, Machado ve ark. (20) buffaloda arteria reticularis'in arteria gastrica sinistra'dan; Alsafy (15) keçide arteria ruminalis sinistra'dan; Mohamed ve ark. (10) koyunda arteria gastrica sinistra'dan veya arteria ruminalis sinistra'dan orijin aldıklarını rapor etmişlerdir.

Sonuç olarak; araştırma materyali olarak kullanılan hasmer koyununda, arteria celiaca'nın aorta abdominalis'teki başlangıcından, organları vaskülarize eden dallarına kadar diğer koyun ırklarına göre birtakım farklılıklar saptandı. Tespit edilen farklılıkların ırk özelliğinden kaynaklanabileceği kanısına varıldı.

\section{Çıkar Çatışması}

Yazarlar, çıkar çatışması olmadığını beyan eder.

\section{KAYNAKLAR}

1. Akçapınar H., Tekin ME., Kadak R., Akmaz A., Müftüoğlu Ş., 1992. Merinos, Alman Siyah Başlı Etçi $x$ Merinos, Hampshire Down $x$ Merinos ve
Lincoln x Merinos (F1) kuzularının büyüme, besi ve karkas özellikleri. Hayvancılık Araştırma Derg, 2, 18-23.

2. Tekin ME., Akçapınar H., 1992. Türk Merinosu ve Lincoln x Türk Merinosu (F1) melezi kuzuların büyüme, besi ve karkas özelliklerinin karşılaştırılması. II. Besi özellikleri. Lalahan Hayvancılık Araştırma Derg, 32, 28-39.

3. Kaymakçı M., Taşkın T., 2008. Türkiye koyunculuğunda melezleme çalışmaları. Hayvansal Üretim, 49, 43-51.

4. Özüdoğru Z., Özdemir D., Balkaya H., Kara H., 2019. Konya Merinosunun arteria celiaca'sı ve dalları üzerine macroanatomik bir çalışma. Atatürk Üniv Vet Bil Derg, 14, 45-51.

5. Karadağ H., 1988. The anatomical comparative study on the celiac artery and its branches in Akkaraman sheep and ordinary goat. J Vet Sci, 12, 196-204.

6. Karmona KH., Kovachev G., 1985. Variability of the celiac artery and its branches in sheep. Vet Med Nauki, 22, 31-37.

7. Türkmenoğlu ì., 1996. Akkaraman Koyunu ve Ankara Keçisinde karın boşluğu arterleri üzerinde karşılaştırmalı makroanatomik araştırmalar. Selçuk Üniversitesi. Sağlık Bilimleri Enstitüsü, Türkiye.

8. Langenfeld M., Pastea E., 1977. Anatomical variants of the celiac artery in the sheep, with special reference to the celiomesenteric arterial trunk. Anat Anz, 142, 168-174.

9. Mohamed R., Adogwa A., Driscol M., Rampersad S., 2016. Arterial supply of the stomach of the barbados black belly sheep in Trinidad. Int J Vet Sci, 5, 142-147.

10. Balkaya H., Özüdoğru Z., Özdemir D., 2017. Güney Karaman koyununda arteria celiaca ve dallarının makroanatomik yapısı. I. Uluslararası Türkiye Veteriner Anatomi Kongresi \& X. Ulusal Veteriner Anatomi Kongresi 13-16 Eylül 2017, Sandıklı/Türkiye.

11. Boccaletti D., Borelli V., 1981. Ramification of the celiac artery in sheep of Corriedale race. Belo 
Horizonte, Minas Gerais, Brasil, Socied Brasil de Anat, pp: 47- 48.

12. Venkatesh B., Purushotham G., Pramod Kumar D., Raghavender KBP., 2018. Anatomical distribution of the hepatic artery in sheep. J Pharm and Phytochem, 7, 2529-2533.

13. Venkatesh B., Purushotham G., Pramod Kumar D., Raghavender KBP., 2018. Anatomical studies on the cystic artery of sheep (Ovis aries). The Pharma Innov J, 7, 650-655.

14. Koç O., Acar K., Özbek O., Güler I., Sarıtaş K., Erdem TB., Solak Y., Toy H., Küçükapan A., Özbek S., Gaipov A., Haznedaroğlu iC., 2016. Splenic artery embolization with Ankaferd blood stopper in a sheep model. Diagn Interv Radiol, 22 (4), 354-357.

15. Alsafy M., 2009. Celiac trunk and the variability of its branches in goats. J App Biolog Sci, 3, 6570.

16. El Gendy SAA., 2007. Surgical anatomical approach of the abdomen in the goat. PhD thesis, Faculty of Veterinary Medicine, Alexandria University, Alexandria.

17. Mohamed R., Adam Z., Gad M., 2017. Arterial supply of the stomach of the Egyptian native goat. J Adv Vet Anim Res, 4, 80-87.

18. Youssef GA., 1991. Some anatomical studies on the coeliac, cranial mesenteric and caudal mesenteric arteries of goat. PhD Thesis, Faculty of Vet. Med. Moshtohor, Zagazig University; Benha, Egypt.

19. Maala C., Reyes EV., Ducusin RJ., 1988. Some contributions to the anatomy of the arterial supply to the stom-ach of the goat. Philipp. J Vet Med, 25, 47-52.

20. Machado F., Miglino A., Didio A., Oliveira Borges
C., 2002. The arterial supply of buffalo stomachs (Bubalus Bubalis). Buffalo J, 18, 257-265.

21. McCarthy PH., 1984. Transruminal palpation of abdominal arteries of the permanently fistulated dairy cow. Am J Vet Res, 45, 1632-1937.

22. Yousefi MH., 2016. Ramification of celiac artery in the pine marten (Martes martes). Iranian J Vet Sci and Tech, 8, 60-65.

23. Kuru N., 2016. Macroanatomic investigations of the course and distribution of the celiac artery in the New Zealand rabbit. Van Vet Journal, 27, 117-122.

24. Perez W., Vazques N., Ungerfeld R., 2016. Arterial vasularization of the gastrointestinal tract of the pampas deer. Anat Histol Embryol, 45, 240-245.

25. Anonymous., 2012. Nomina Anatomica Veterinaria, 5th. Ed. Copyright by the World Association of Veterinary Anatomists.

26. Dursun N., 2008. Veteriner Anatomi II. 12. Baskı. Medisan Yayınevi, 128-163, Ankara.

27. Dyce KM., Sack WO., Wensing CJG., 1987. Text Book of Veterinary Anatomy. W.B. Saunders Company, Philadelphia.

28. Smuts MMS., Bezuidenhout AJ., 1987. Anatomy of the Dromedary. Clarendon press, pp: 156-170, 175-177, Oxford.

29. Getty R., 1975. Sisson and Grossman's The Anatomy of the Domestic Animals. Vol.2, Fifth ed., W. B. Saunders Company, Philedephia, London.

30. Nayar KN., Singh MG., Singh Y., Singh P., Singh R., 1983. Comparative arteriographic anatomy of the abdominal viscera and lumbar region in goats, dogs, pigs and rabbits. Indian j Anim Sci, $53,1310-1314$ 